Portland State University

PDXScholar

$11-1-1998$

\title{
Simple Model for Linear and Nonlinear Mixing at Unstable Fluid Interfaces with Variable Acceleration
}

John D. Ramshaw

Portland State University, jdramshaw@yahoo.com

Follow this and additional works at: https://pdxscholar.library.pdx.edu/phy_fac

Part of the Physics Commons

Let us know how access to this document benefits you.

Citation Details

J.D. Ramshaw, "Simple model for linear and nonlinear mixing at unstable fluid interfaces with variable acceleration," Phys. Rev. E 58, 5834 (1998).

This Article is brought to you for free and open access. It has been accepted for inclusion in Physics Faculty Publications and Presentations by an authorized administrator of PDXScholar. Please contact us if we can make this document more accessible: pdxscholar@pdx.edu. 


\title{
Simple model for linear and nonlinear mixing at unstable fluid interfaces with variable acceleration
}

\author{
John D. Ramshaw \\ Lawrence Livermore National Laboratory, University of California, P.O. Box 808, L-097, Livermore, California 94551
}

(Received 1 April 1998)

\begin{abstract}
A simple model is described for predicting the time evolution of the half-width $h$ of a mixing layer between two initially separated immiscible fluids of different density subjected to an arbitrary time-dependent variable acceleration history $a(t)$. The model is based on a heuristic expression for the kinetic energy per unit area of the mixing layer. This expression is based on that for the kinetic energy of a linearly perturbed interface, but with a dynamically renormalized wavelength which becomes proportional to $h$ in the nonlinear regime. An equation of motion for $h$ is then derived from Lagrange's equations. This model reproduces the known linear growth rates of the Rayleigh-Taylor (RT) and Richtmyer-Meshkov (RM) instabilities, as well as the nonlinear RT growth law $h=\alpha A a t^{2}$ for constant $a$ (where $A$ is the Atwood number) and the nonlinear RM growth law $h \sim t^{\theta}$ for impulsive $a$, where $\alpha$ and $\theta$ depend on the rate of kinetic energy dissipation. In the case of zero dissipation, $\theta=2 / 3$ in agreement with elementary scaling arguments. A conservative numerical scheme is proposed to solve the model equations, and is used to perform calculations that agree well with published experimental mixing data for four different acceleration histories. [S1063-651X(98)13411-6]
\end{abstract}

PACS number(s): 47.20.Bp, 47.20.Ma, 47.27.-i, 47.55.Kf

\section{INTRODUCTION}

There is considerable current interest in unstable fluid interfaces, particularly those driven by the normal acceleration of adjacent fluid layers with different densities. The initial unperturbed interface is assumed to be planar, and we restrict attention to incompressible immiscible fluids with negligible surface tension. The degree to which the two fluids are mixed together by an instability may be characterized by the half-width $h(t)$ of the mixing layer as a function of the time $t$. This half-width is usually defined as the visual penetration depth of the lighter fluid into the heavier one. Alternative definitions of $h$ will differ in numerical value but should be of the same order of magnitude. For theoretical purposes it might be preferable to define $h$ as the volume per unit area transported (exchanged) across the initial interface. This quantity is inherently symmetrical between the two fluids, but unfortunately is difficult to determine experimentally.

The two classical instabilities of this type are the Rayleigh-Taylor (RT) [1] and Richtmyer-Meshkov (RM) $[2,3]$ instabilities, which respectively correspond to the opposite limiting cases of constant and impulsive acceleration. These instabilities are respectively defined by $a(t)=a_{0}$ $=$ const and $a(t)=\Delta v \delta(t)$, where $a(t)$ is the timedependent acceleration of the interface in the normal direction. In the linear regime, $h$ may be identified with the amplitude of a small sinusoidal perturbation of wavelength $\lambda$ $=2 \pi / k$. The time evolution of $h(t)$ may then be determined by a conventional linear stability analysis, which yields [1]

$$
\ddot{h}=k A a h,
$$

where $\dot{q}=d q / d t$ for any $q, A=\left(\rho_{2}-\rho_{1}\right) /\left(\rho_{2}+\rho_{1}\right)$ is the Atwood number, and $\rho_{1}, \rho_{2}$ are the densities of the two adjacent incompressible fluids, which are labeled so that a positive acceleration $a(t)$ is directed from fluid 1 into fluid 2 .
[The acceleration $a(t)$ is of course equivalent to an artificial external body force $g(t)$ in the opposite direction, and vice versa.] For an initially quiescent interface with $\dot{h}=0$ at $t$ $=0-$, it follows that $h=\frac{1}{2} h_{0}\left(e^{r t}+e^{-r t}\right)$ in the RT case [where $h_{0}=h(0)>0$ and $r^{2}=k A a_{0}$ ] and $h=h_{0}(1$ $+k A \Delta v t)$ in the RM case. Thus the RT case exhibits exponential growth, but only when $A a_{0}>0$, whereas the RM case exhibits linear growth regardless of the sign of $A \Delta v$. Notice that negative values of $h$ must be allowed in order to describe the RT stable cases $A a_{0}<0$ and $A \Delta v<0$. This merely indicates that the initial displacement has suffered a reversal in direction, in which case the half-width of the mixing layer may be identified with $|h|$.

In the late-time fully nonlinear regime, $h(t)$ is believed to obey simple scaling laws for both the RT and RM instabilities. The late-time scaling law for the RT instability is given by $[4-6]$

$$
h=\alpha A a_{0} t^{2}
$$

for $A a_{0}>0$, where the dimensionless coefficient $\alpha$ is of order 0.05 and is nearly independent of $A$. This time dependence follows from a simple dimensional argument [4], but the fact that $h$ remains nearly linear in $A$ even in the nonlinear regime is noteworthy, as this does not follow from dimensional considerations alone.

The corresponding late-time scaling law for the RM instability is somewhat more subtle, and takes its simplest form in the inviscid case where there is no dissipation of kinetic energy into thermal energy. In this case the kinetic energy per unit area $K$ deposited by the impulsive acceleration is conserved for $t>0$ and becomes available for use in constructing a similarity variable. The same type of dimensional argument used by Taylor to determine the scaling law for an expanding blast wave [7] then leads immediately [8] to the RM scaling law 


$$
h=\beta\left(\frac{K}{\bar{\rho}}\right)^{1 / 3} t^{2 / 3}
$$

where $\bar{\rho}=\frac{1}{2}\left(\rho_{1}+\rho_{2}\right)$ and $\beta$ is another dimensionless coefficient, which may, however, depend upon $A$. This scaling law is also confirmed by other arguments $[9,10]$ which further indicate that the time exponent of $2 / 3$ is shifted to smaller values by nonzero dissipation. The RM scaling law then takes the form $h \sim t^{\theta}[9-11]$, where the exponent $\theta \leqslant 2 / 3$ is somewhat uncertain. Experiments and numerical simulations to date have yielded a fairly wide range of values for $\theta$ (see [11], and references cited therein), which mostly tend to lie below 2/3 consistent with the effects of dissipation. (It also seems likely that some of these previous studies did not reach late enough times to see the asymptotic limiting behavior, and this probably accounts for some of the scatter in the reported values of $\theta$.) Based on Eq. (3), however, it seems quite certain that $\theta=2 / 3$ in an ideal incompressible system with zero dissipation. It should be possible to confirm this by direct numerical simulations carried to sufficiently late times, provided that care is taken to minimize the effects of numerical dissipation. It is of course not surprising that the RM case should be more sensitive to dissipation than the RT case, since the constant acceleration provides a continual supply of kinetic energy in the latter which is absent in the former.

Use of the ideal RM scaling law requires knowledge of the kinetic energy per unit area $K$ deposited by the impulsive acceleration. This energy may readily be evaluated in the linear approximation, with the result $[10,12,13]$

$$
K=\frac{1}{2} \bar{\rho} k\left(A \Delta v h_{0}\right)^{2}
$$

which depends on the amplitude and wavelength of the initial perturbation as well as the strength of the impulse. It follows that even at late times, the RM instability retains memory of the initial conditions through $K$, whereas the RT instability is believed to lose all memory of the initial conditions at late times [4]. This difference is a further reflection of the fact that the RT instability is driven by a continuous source of kinetic energy which is absent in the RM case.

The pure limiting cases of RT and RM instability are rarely encountered in practical situations, where the acceleration $a(t)$ usually exhibits a more complicated time dependence (see $[6,14,15]$, and references cited therein). Our purpose here is to present a simple model for predicting $h(t)$ for arbitrary $a(t)$. This model is based on a heuristic expression for the kinetic energy per unit area of the mixing layer. This expression has the same form as that for the kinetic energy of a linearly perturbed interface, but with the perturbation wavelength $\lambda=2 \pi / k$ replaced by a dynamically renormalized wavelength $\lambda(t)$ which is postulated to become proportional to $h$ in the nonlinear regime. An equation of motion for $h$ is then derived from Lagrange's equations, with the inclusion of an additional generalized force term to represent the effects of dissipation [16]. In the special cases of constant and impulsive acceleration, this model correctly reproduces all of the known growth behavior and scaling laws summarized above for both the RT and RM instabilities in both the linear and nonlinear regimes. This lends some confidence in its use for arbitrary $a(t)$. We have also verified that the model accurately represents the variable-acceleration experimental data of Dimonte and Schneider [6], but this is not a very stringent test for reasons to be discussed in Sec. V. Further applications to a wider range of variable-acceleration experimental data and direct numerical simulations will be required to obtain a better assessment of the overall accuracy and utility of the model for arbitrary $a(t)$.

\section{CONSTRUCTION OF THE MODEL}

We consider a flat slab of large but finite thickness composed of two adjacent incompressible fluid layers. The unperturbed interface between the fluids is located at $z=Z(t)$ in a Cartesian coordinate system $(x, y, z)$. Fluid 1 has density $\rho_{1}$ and occupies the region $Z(t)-\Delta Z_{1}<z<Z(t)$, while fluid 2 has density $\rho_{2}$ and occupies the region $Z(t)<z<Z(t)$ $+\Delta Z_{2}$. The unperturbed velocity of both fluids is then simply $\dot{Z}$ in the $z$ direction, and the acceleration of the slab is $a(t)=\ddot{Z}$. Now suppose that the interface location is perturbed to $z=Z(t)+h(t) \cos k x$, where $|h| k \ll 1$ and $|h|$ $\ll \Delta Z_{i}$. We require the resulting potential flow field $\mathbf{u}$ $=\nabla \phi$ to first order in $h$. In this linear approximation the boundary conditions on the velocity potential $\phi$ are simply $\partial \phi / \partial z=\dot{Z}+\dot{h} \cos k x$ at $z=Z$ and $\partial \phi / \partial z \rightarrow \dot{Z}$ for $|z-Z|$ $\gg|h|$. The solution to Laplace's equation under these conditions is readily found by separation of variables, and is given by

$$
\phi=\dot{Z} z \mp \frac{\dot{h}}{k} \cos k x \exp [\mp k(z-Z)]
$$

where the upper sign applies for $z>Z+h \cos k x$ and the lower sign for $z<Z+h \cos k x$. The total kinetic energy per unit area of the slab is then given by $T$ $=\frac{1}{2} \int d x d y d z \rho|\nabla \phi|^{2} / \int d x d y$. Since $T$ is quadratic in $\phi$, it must be evaluated to second order in $h$ and/or $\dot{h}$ to describe the linear regime. For this purpose it is essential to include the second-order effects of the perturbation on the $z$ integration limits. However, it is unnecessary to evaluate $\phi$ itself to second order, since the linearized interface dynamics is completely determined by the linear approximation to $\phi[1]$. The second-order correction to $\phi$ therefore cannot contribute to $T$ to second order, and this has been directly confirmed by a more detailed analysis. The required integrations are straightforward, and the resulting second-order expression for $T$ is given by

$$
T=\frac{1}{2 k} \bar{\rho} \dot{h}^{2}-\frac{1}{2} \Delta \rho h \dot{h} \dot{Z}+\frac{1}{2} M \dot{Z}^{2},
$$

where $M=\rho_{1} \Delta Z_{1}+\rho_{2} \Delta Z_{2}$ and $\Delta \rho=\rho_{2}-\rho_{1}=2 \bar{\rho} A$. The first term in Eq. (6) represents the kinetic energy in a Galilean coordinate frame moving with velocity $\dot{Z}$. This is just the intrinsic kinetic energy of the moving perturbed interface, which is therefore given by

$$
K=\frac{1}{2 k} \bar{\rho} \dot{h}^{2} .
$$


This expression reduces to Eq. (4) in the impulsive case, where $\dot{h}=k A \Delta v h_{0}$ for $t>0$.

Equation (6) will be used to obtain the dynamical evolution of the interface from Lagrange's equations [16] in terms of the generalized coordinates $h$ and $Z$ and their time derivatives $\dot{h}$ and $\dot{Z}$. For this purpose we must also consider the potential energy $V$ associated with whatever external forces (presumed conservative) are employed to produce the acceleration $a(t)$. But these forces are applied at the outer surfaces of the slab, so they are independent of $h$. It follows that $V=V(Z)$ is also independent of $h$ and will therefore not contribute to the Lagrange equation of motion for $h$ [16]. Since this is the only equation of motion we shall consider, $V(Z)$ can henceforth be ignored and the Lagrangian $L$ can simply be identified with $T$.

We first verify that this approach correctly reproduces the known linearized equation of motion for $h$, namely Eq. (1). In the absence of dissipation, Lagrange's equation for $h$ is simply [16]

$$
\frac{d}{d t}\left(\frac{\partial T}{\partial \dot{h}}\right)=\frac{\partial T}{\partial h} .
$$

The required partial derivatives of $T$ are easily evaluated from Eq. (6), with the results $\partial T / \partial \dot{h}=(\bar{\rho} / k) \dot{h}-\frac{1}{2} \Delta \rho h \dot{Z}$ and $\partial T / \partial h=-\frac{1}{2} \Delta \rho \dot{h} \dot{Z}$. Combining these derivatives with Eq. (8), we immediately obtain Eq. (1). Thus the linear regime is properly described by Eqs. (6) and (8), so we may now direct our attention to the late-time fully nonlinear regime.

Equation (6) is based on a linearized analysis, so it clearly no longer strictly applies in the nonlinear regime. However, there are nevertheless heuristic reasons for suspecting that an appropriate reinterpretation of the linear analysis may retain some validity in the nonlinear regime as well. We observe that this problem contains no natural length scale (in the limit of large $\Delta Z_{i}$ ), so there is no objective basis for describing the mixing layer as being either thick or thin. An observer's subjective impression of the thickness of the mixing layer is determined entirely by the distance from which it is viewed. The mixing layer will always look thin when viewed from a sufficiently distant vantage point. That is to say, from far away the interface will always appear to be only slightly perturbed, with an apparent perturbation amplitude of order $|h|$. Of course, the perturbation will now be irregular rather than sinusoidal, and the apparent transverse length scale of the irregularities will also be of order $|h|$. This length scale plays the role of the effective wavelength of the perturbation. These heuristic considerations suggest that, at least in some rough scaling sense, the mixing layer may be expected to behave as though it always remains in the linear regime, but with a time-dependent perturbation wavelength $\lambda(t)$ which is continuously dynamically renormalized to a value of order $|h(t)|$. We therefore adopt the working hypothesis that Eq. (6) remains valid even in the fully nonlinear regime with $\lambda$ $=2 \pi / k$ replaced by $\lambda(t)=b|h(t)|$, where $b$ is a dimensionless coefficient of order unity which remains to be determined. This hypothesis will be referred to as the wavelength renormalization hypothesis (WRH), the consequences of which will now be explored.
Similar ideas have previously been discussed by other authors. In particular, the above plausibility argument for the WRH is somewhat reminiscent of the motivation for the superposition approximation of Glimm et al. [17], and a similar linear relation between $\lambda$ and $h$ has previously been invoked in the RT context by Youngs [4] and Mikaelian $[18,19]$, in the RM context by Dimonte and co-workers $[11,20]$, and in both contexts by Alon et al. [21]. However, it is essential to realize that this relation does not lead to unique results in and of itself. In particular, differential equations valid for constant $\lambda$, such as Eq. (1), give no indication as to whether factors involving $\lambda(t)$ should appear inside or outside of time derivatives. The manner in which the relation $\lambda(t)=b|h(t)|$ is introduced is therefore critical. By introducing this relation into $T$ and then using Lagrange's equations to determine the time evolution of $h(t)$, we automatically preserve the essential property of energy conservation, which of course is the basis for Eq. (3). Other ways of introducing the relation $\lambda(t)=b|h(t)|$ may lead to results inconsistent with Eq. (3) in the RM case, such as $h \sim \sqrt{t}[11,20]$.

It is convenient to combine the linear and nonlinear cases by letting $\lambda=2 \pi / k=\lambda(|h|)$. Equation (6) then becomes

$$
T=\frac{\bar{\rho}}{4 \pi} \lambda(|h|) \dot{h}^{2}-\frac{1}{2} \Delta \rho h \dot{h} \dot{Z}+\frac{1}{2} M \dot{Z}^{2},
$$

while Eq. (7) becomes

$$
K=\frac{\bar{\rho}}{4 \pi} \lambda(|h|) \dot{h}^{2} .
$$

These expressions now encompass both constant $\lambda$ and the WRH $(\lambda=b|h|)$ as special cases. Of course, a transition between these two cases must be made at some appropriate intermediate value (or over some range of values) of $|h|$. This transition will be addressed in Sec. IV.

As previously discussed, it will also be necessary to allow for energy dissipation in order to realistically represent the nonlinear regime. This can be done by introducing an additional generalized force $Q$ into Eq. (8) to represent the effects of dissipation [16]:

$$
\frac{d}{d t}\left(\frac{\partial T}{\partial \dot{h}}\right)=\frac{\partial T}{\partial h}+Q .
$$

We shall assume that the dissipation rate of kinetic energy in the nonlinear regime is controlled by the large scale motions and is consequently independent of molecular viscosity, just as it is in turbulence [22]. This implies that the dissipative force $Q$ can be expressed entirely in terms of $\bar{\rho}, h$, and $\dot{h}$. Since $h$ is a distance coordinate, $Q$ has the units of force per unit area or energy per unit volume. Thus $Q$ must be of order $\bar{\rho} \dot{h}^{2}$, and in order to be purely dissipative in nature it must have the opposite sign from $\dot{h}$. It follows that $Q$ must be of the form

$$
Q=-c \bar{\rho}|\dot{h}| \dot{h},
$$

where $c \geqslant 0$ is a new dimensionless coefficient of order unity which may depend upon $A$. 
Equations (9), (11), and (12) may now be combined to obtain a general equation of motion for $h$. Evaluating the required partial derivatives of $T$ from Eq. (9), we readily obtain

$$
\lambda \ddot{h}+\frac{1}{2} \dot{\lambda} \dot{h}+2 \pi c|\dot{h}| \dot{h}-2 \pi A a h=0,
$$

where use has been made of the fact that $\dot{\lambda}=(\partial \lambda / \partial h) \dot{h}$ $=(d \lambda / d|h|)(h /|h|) \dot{h}$. Equation (13) is the fundamental dynamical evolution equation of the model. It is a second-order ordinary differential equation which determines $h(t)$ for an arbitrary given $a(t)$. However, the model is not yet complete because we have not yet specified the values of the model coefficients $b$ and $c$, or the functional form of $\lambda(|h|)$ required to provide a suitable transition between the linear and nonlinear regimes. These quantities will be determined in Secs. III and IV below.

In the linear regime with zero dissipation, $\lambda$ is constant with its initial value $\lambda_{0}$ so $\dot{\lambda}=c=0$ and Eq. (13) reduces to Eq. (1). In the nonlinear (WRH) regime we have $\lambda=b|h|$ and $\dot{\lambda}=b(h /|h|) \dot{h}$, and Eq. (13) becomes

$$
b|h| \ddot{h}+\frac{b h}{2|h|} \dot{h}^{2}+2 \pi c|\dot{h}| \dot{h}-2 \pi A a h=0 .
$$

In terms of $v \equiv \dot{h}$, Eq. (14) takes the form

$$
\dot{v}=\frac{2 \pi h}{b|h|} A a-\left(\frac{v}{2 h}+\frac{2 \pi c}{b}\left|\frac{v}{h}\right|\right) v .
$$

This equation is reminiscent of previous simple mixing-layer models based on bubble-rise dynamics $[6,11,21,23,24]$, in which $v$ represents the bubble velocity and the terms proportional to $a$ and $v$ are respectively interpreted as buoyancy and drag forces, with the coefficients adjusted to allow for such additional effects as added mass and entrainment. Such models have indeed met with some success in correlating experimental data [6], but have previously seemed rather too ad hoc and approximate to be taken very seriously. It is therefore noteworthy that the present development leads to a similar formulation based on an entirely different and considerably more general approach in which bubble dynamics and the ingredients thereof (buoyancy, drag, added mass, etc.) play no explicit role. It is tempting to interpret this as an indication that models of this type may be somewhat better founded than they first appear. However, it should not be assumed that all such models are essentially equivalent or interchangeable, as they may exhibit qualitative as well as quantitative differences. In this regard, we note in particular that the term proportional to $v$ in Eq. (15) cannot in fact be interpreted as a pure drag force, since it does not always act in opposition to $v$ and does not vanish in the limit of zero dissipation $(c=0)$. Indeed, the remaining term $v^{2} / 2 h$ is purely conservative in nature, in spite of its appearance, since energy is conserved when $c=0$. We further note that the precise placement of the absolute values in Eqs. (13)(15) is critical in cases where $h$ and/or $\dot{h}$ become negative.
It is instructive to examine the time evolution of $K$, which may be obtained by differentiating Eq. (10) and combining the result with Eq. (13). We thereby obtain

$$
\dot{K}=\bar{\rho} A a h \dot{h}-c \bar{\rho}|\dot{h}|^{3},
$$

which shows that the force $Q$ dissipates kinetic energy at the positive definite rate $c \bar{\rho}|\dot{h}|^{3}$. In the RT case with $a=a_{0}$ $=$ const, the constant acceleration is equivalent to a potential energy per unit area $U=-\frac{1}{2} \bar{\rho} A a_{0} h^{2}$. Equation (16) may then be written in the alternative form $\dot{E}=-c \bar{\rho}|\dot{h}|^{3}$, where $E=K+U$. Thus $\dot{E}=0$ when $c=0$, so the total energy of the mixing layer is conserved in the absence of dissipation, just as it should be.

\section{ASYMPTOTIC LATE-TIME RT AND RM BEHAVIOR}

We have already verified that the model correctly reduces to the linear growth law of Eq. (1) in the linear regime. We now proceed to verify that it also correctly reproduces the known late-time RT and RM scaling laws in the nonlinear regime. In doing so, we shall automatically obtain relations which uniquely determine the model coefficients $b$ and $c$ in terms of the experimentally accessible scaling parameters $\alpha$ and $\theta$.

We first consider the RT case, in which $a(t)=a_{0}$ $=$ const, $A a_{0}>0$, and both $h$ and $\dot{h}$ remain positive for all time. Equation (14) then becomes

$$
h \ddot{h}+\left(\frac{1}{2}+\frac{2 \pi c}{b}\right) \dot{h}^{2}-\frac{2 \pi A a_{0}}{b} h=0 .
$$

One readily verifies by direct substitution that the RT scaling law $h=\alpha A a_{0} t^{2}$ satisfies Eq. (17) provided that

$$
\alpha=\frac{\pi}{2 b+4 \pi c} .
$$

This shows that $b$ would have the value $b=\pi / 2 \alpha \cong 10 \pi$ $\cong 30$ in an ideal system with zero dissipation. It follows that $|h| \ll \lambda$ even in the nonlinear regime, which further supports the idea that the system will continue to behave in an essentially linear manner in this regime. The WRH is thus internally self-consistent in this sense.

We now consider the RM case, in which $a(t)=0$ for $t$ $>0$. Now $h$ and $\dot{h}$ have the same sign at late times, which may be taken as positive without loss of generality. The absolute value signs in Eq. (14) can then be omitted, so that

$$
h \ddot{h}+\left(\frac{1}{2}+\frac{2 \pi c}{b}\right) \dot{h}^{2}=0 .
$$

This equation is easily solved by elementary manipulations, with the result

$$
h(t)=h_{1}\left[1+p\left(\dot{h}_{1} / h_{1}\right)\left(t-t_{1}\right)\right]^{1 / p},
$$

where $h_{1}$ and $\dot{h}_{1}$ are the values of $h$ and $\dot{h}$ at $t=t_{1}$, and

$$
p=\frac{3}{2}+\frac{2 \pi c}{b}=\frac{3 b+4 \pi c}{2 b} .
$$


It follows that $h \sim t^{\theta}$ for large $t$, where $\theta=1 / p$ or

$$
\theta=\frac{2}{3}\left(1+\frac{4 \pi c}{3 b}\right)^{-1}=\frac{2 b}{3 b+4 \pi c} .
$$

This shows that $\theta=2 / 3$ when $c=0$, in agreement with Eq. (3), and it further exhibits the expected reduction in $\theta$ due to dissipation $[9,10]$. [It is noteworthy that Eq. (18) shows that $\alpha$ exhibits a similar reduction, which to our knowledge has not previously been suggested.] Equations (18) and (22) now uniquely determine the model coefficients $b$ and $c$ in terms of the RT scaling parameter $\alpha$ and the RM scaling parameter $\theta$. Inverting these relations, we readily obtain

$$
\begin{gathered}
b=\frac{\pi \theta}{\alpha(2-\theta)}, \\
c=\frac{2-3 \theta}{4 \alpha(2-\theta)} .
\end{gathered}
$$

Equation (20) implies that $\dot{h}=\dot{h}_{1}\left(h_{1} / h\right)^{p-1}$, which combines with Eq. (10) (with $\lambda=b h$ ) to yield $4 \pi K$ $=\bar{\rho} b \dot{h}_{1}^{2} h_{1}\left(h / h_{1}\right)^{3-2 p}$. This may be rewritten in the form

$$
h(t)=\left(\frac{4 \pi K(t)}{\bar{\rho} b}\right)^{1 / 3}\left[\frac{h_{1}}{\dot{h}_{1}}+p\left(t-t_{1}\right)\right]^{2 / 3},
$$

which shows that Eq. (3) is not in fact restricted to the inviscid case as previously presumed, but is actually valid in general with $\beta=(4 \pi / b)^{1 / 3} p^{2 / 3}$ or

$$
\beta=\frac{2}{\theta}\left[\alpha\left(1-\frac{\theta}{2}\right)\right]^{1 / 3} .
$$

This provides a new theoretical relation between $\alpha, \beta$, and $\theta$. Of course, $K$ decays with time when $c \neq 0$ so Eq. (3) is not particularly useful in that case, but it is remarkable that it nevertheless remains valid. In the inviscid case, $\theta=2 / 3$ and Eq. (26) reduces to $\beta=(18 \alpha)^{1 / 3}$. This provides a new and to our knowledge previously unsuspected theoretical relation between $\alpha$ and $\beta$ in the absence of dissipation.

\section{TRANSITION BETWEEN THE LINEAR AND NONLINEAR REGIMES}

We have seen that Eq. (13) correctly reproduces the known behavior of the RT and RM mixing layers in both the linear and nonlinear regimes. We now return to the question of how to prescribe a suitable transition between these regimes. The obvious choice would be to effect the transition at the point where $b|h|$ becomes equal to the initial perturbation wavelength $\lambda_{0}$. This may be done by writing $\lambda$ $=\max \left(\lambda_{0}, b|h|\right)$. According to Eq. (23), $b \cong 25$ for reasonable values of $\theta$, so the resulting transition occurs at $|h|$ $\cong 0.04 \lambda_{0}$. This seems much too early, since one would intuitively expect the linear regime to persist until $|h| \sim \lambda_{0}$. The transition can be delayed to $|h|=m \lambda_{0}$ (where $m \sim 1$ may be set at the user's discretion) by writing

$$
\lambda=\max \left[\lambda_{0}, b|h|+(1-m b) \lambda_{0}\right],
$$

which does not change the late-time asymptotic behavior. However, this is clearly still a primitive and highly oversimplified transition rule which should not be expected to be very accurate. It would be of obvious interest to consider more elaborate prescriptions in which the linear and fully nonlinear regimes are continuously connected by a weakly nonlinear transitional regime which extends over a finite range of $|h|$. Analytical expressions which might serve as a basis for such a prescription have recently been presented by Mikaelian [25]. It should be noted, however, that when $\lambda_{0}$ is very small the transition to the nonlinear regime occurs so quickly that the detailed manner in which it does so becomes relatively unimportant.

The model is now complete. The dynamical evolution of the mixing layer is determined by Eq. (13) for arbitrary $a(t)$, with $b$ and $c$ given by Eqs. (23) and (24) and $\lambda$ given by Eq. (27). The model provides a unified description of the time evolution of $h$ in both the linear and nonlinear regimes, with an automatic transition between them at $|h|=m \lambda_{0}$. Since the form of the dissipation term in Eq. (13) was obtained from inherently nonlinear considerations, this term should be switched off in the linear regime by setting $c=0$ for $|h|$ $<m \lambda_{0}$. The model requires values of $\alpha, \theta, \lambda_{0}, m$, and $a(t)$ as input data, and values of $h(0) \equiv h_{0}$ and $\dot{h}(0) \equiv \dot{h}_{0}$ at $t$ $=0$ as initial conditions. In problems that start out in the linear regime with an impulsive acceleration $a(t)=\Delta v \delta(t)$ at $t=0$, the effect of the impulse is to increment the initial value of $\dot{h}$ by an amount $\dot{h}(0+)-\dot{h}(0-)=2 \pi A \Delta v h_{0} / \lambda_{0}$. It is unnecessary to explicitly include the impulse in $a(t)$ if $\dot{h}_{0}$ is identified with $\dot{h}(0+)$ rather than $\dot{h}(0-)$.

In the special case of zero dissipation $(c=0)$ and with the linear-to-nonlinear transition defined by $\lambda=\lambda_{0}+b|h|$, Eq. (13) reduces to an earlier unpublished model independently derived by Stry [26]. In this model the linear-to-nonlinear transition occurs even earlier than with $\lambda=\max \left(\lambda_{0}, b|h|\right)$. This seems undesirable in general, but again becomes immaterial when $\lambda_{0}$ is very small.

\section{NUMERICAL SOLUTION OF THE MODEL EQUATIONS}

In general it will be necessary to solve Eq. (13) numerically to obtain solutions for arbitrary acceleration histories $a(t)$. For numerical purposes it is convenient to replace the second-order Eq. (13) by an equivalent system of two coupled first-order equations. It is further convenient to introduce the new variable $w=\sqrt{\lambda} \dot{h}$, in terms of which $4 \pi K$ $=\bar{\rho} w^{2}$ becomes strictly quadratic. One then readily verifies that Eq. (13) is equivalent to the first-order system

$$
\begin{gathered}
\dot{h}=\frac{w}{\sqrt{\lambda}}, \\
\dot{w}=\frac{2 \pi A a h}{\sqrt{\lambda}}-\frac{2 \pi c|w| w}{\lambda^{3 / 2}} .
\end{gathered}
$$

We have already seen that these equations conserve energy when $a(t)=a_{0}=$ const and $c=0$. It is clearly desirable to use a numerical scheme which preserves this important conser- 


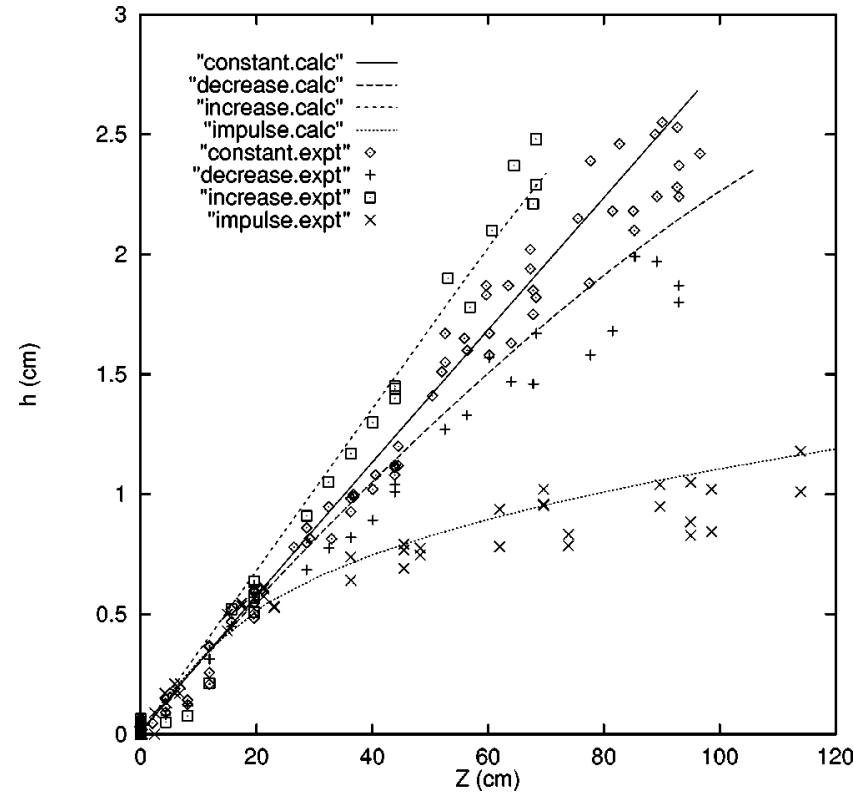

FIG. 1. Comparison of model calculations with experimental data for four different acceleration histories.

vation property. One readily verifies that the following scheme meets this requirement:

$$
\begin{gathered}
\frac{h^{n+1}-h^{n}}{\Delta t}=\frac{w^{n+1}+w^{n}}{2 \sqrt{\lambda^{n}}} \\
\frac{w^{n+1}-w^{n}}{\Delta t}=\frac{\pi A a^{n}}{\sqrt{\lambda^{n}}}\left(h^{n+1}+h^{n}\right)-\frac{2 \pi c\left|w^{n}\right| w^{n+1}}{\left(\lambda^{n}\right)^{3 / 2}} .
\end{gathered}
$$

Here $q^{n}$ denotes the numerical approximation to the quantity $q$ at time $t^{n}$, and $\Delta t=t^{n+1}-t^{n}$ is the time step. The natural time scale $\tau$ in these equations is given by $1 / \tau^{2}$ $=2 \pi|A a| / \lambda$, and it is of course necessary to restrict $\Delta t \ll \tau$ to obtain an accurate solution. Notice that the dissipative term has been treated in a linearly implicit manner to avoid a corresponding stability restriction on $\Delta t$. Equations (30) and (31) constitute a linear system of two equations in the two unknown quantities $h^{n+1}$ and $w^{n+1}$. These equations are easily solved to advance the system in time.

We have used the scheme of Eqs. (30) and (31) to generate numerical solutions corresponding to the variableacceleration experimental data of Dimonte and Schneider (DS) [6], using the DS parameter values $\alpha=0.061$ and $\theta$ $=0.37$. The acceleration profiles $a(t)$ of the four cases studied by DS were approximated by piecewise linear profiles. The interface was initially flat and glassy in these experiments, so very small values of $\lambda_{0}$ and $h_{0}$ were used. The model consequently enters the nonlinear regime almost immediately. Figure 1 shows calculated (.calc) and experimental (.expt) plots of $h$ vs $Z$ (with $Z=\dot{Z}=0$ at $t=0$ ) for these four cases, using the terminology of DS. The model calculations are indeed in good agreement with the experimental data, but this is not surprising since DS already found that their data were also well represented by a simple bubbledynamics model. As discussed above, models of this type become essentially equivalent to the present model in the nonlinear regime, at least in situations like these experiments where there is no sign reversal of $h$ or $\dot{h}$. More extensive applications to a wider range of variable-acceleration data and simulations will therefore be required to obtain a better assessment of the overall accuracy and utility of the present model for arbitrary $a(t)$. Such studies should ideally include situations in which the linear regime is more important and longer in duration, which can be arranged by the use of larger initial perturbation wavelengths.

It is noteworthy that in these and other calculations where the model immediately enters the nonlinear regime, the solutions are somewhat more sensitive to $h_{0}$ than one might naively expect. The reason is that the RT scaling law of Eq. (2) has an initial slope of zero, so that a relatively small change in $h$ corresponds to a relatively large change in $t$ near $t=0$. An apparently small value of $h_{0}$ can therefore have the effect of introducing an appreciable time shift into $h(t)$, thereby giving the system a significant "head start."

We also performed RM test calculations for both $A \Delta v$ $>0$ and $A \Delta v<0$ in order to verify that the model correctly predicts the reversal of $h$ in the latter case. This reversal indeed occurred with no difficulties, and in both cases the model exhibited a smooth transition between linear growth at early times to $t^{\theta}$ growth at late times. This too is not surprising, since this behavior was built into the model by construction.

Finally, in order to further examine the behavior of the model upon sign reversal of $h$ and/or $\dot{h}$, we performed RT demixing calculations in which the sign of $A a_{0}$ was suddenly changed from positive to negative well into the nonlinear regime. The fluids initially mixed in accordance with the RT growth law of Eq. (2), and then rapidly demixed again when the sign of $A a_{0}$ was reversed. In the case of zero dissipation $(c=0)$, the interface motion overshoots to negative values of $h$, and thereafter the model exhibits undamped nonlinear oscillations in $h$ about $h=0$. These oscillations are simply a nonlinear analog of the stable linear oscillations predicted by Eq. (1) for $A a<0$. This cycle of alternating mixing/demixing persists forever in accordance with energy conservation. However, these oscillations are not structurally stable, as they are rapidly quenched by even very small nonzero values of $c$.

It is encouraging that the model correctly predicts at least the qualitative aspects of demixing. However, the model in its present form is unlikely to provide a satisfactory quantitative description of demixing rates. The reason is that sudden changes in $a$ tend to cause the larger fluid fragments in the mixing layer to break up into smaller fragments, which tends to retard demixing $[15,27]$. This is not surprising, since the smaller fragments will experience larger drag forces. A similar abrupt fragmentation of larger structures is observed in RM experiments in which a second impulsive acceleration follows the first [28]. Effects of this type evidently involve sudden changes in the spectrum of length scales in the mixing layer, and in order to accurately represent them it will probably be necessary to introduce additional variables to carry some of this spectral information. It should also be noted that the phenomenon of demixing will exhibit qualitative differences between miscible and immiscible fluids, to which the present discussion has been restricted. Miscible 
fluids will also undergo irreversible mixing on the atomic level, and this will reduce the degree to which demixing can be accomplished on reversal of $A a$.

\section{CONCLUSION}

We have presented a simple model, embodied in Eqs. (13) and (27), for predicting the time evolution of an incompressible planar fluid mixing layer subjected to an arbitrary timedependent acceleration history. This model correctly reproduces the known growth behavior and scaling laws for both the RT and RM instabilities in both the linear and nonlinear regimes. It is hoped that this model will prove useful in correlating experimental and direct numerical simulation data on mixing at unstable fluid interfaces with variable acceleration. We reemphasize, however, that the model is particularly simplistic in its treatment of demixing effects and the transition between the linear and fully nonlinear regimes. It seems likely that further refinements along the lines discussed in Secs. IV and V will be required to accurately represent these effects in situations where they are important.

The present model is restricted to incompressible fluids. The incompressible RT instability is of reasonably wide in- terest in its own right, whereas the incompressible RM instability is primarily of interest as an approximation to cases in which the impulsive acceleration is produced by the passage of a shock wave through layers of compressible fluid. This approximation then requires one to distinguish between and correct for differences in the preshock and postshock conditions, particularly perturbation amplitude and Atwood number [29-32]. Although we have not considered such corrections here, it should be noted that they are of essential importance for many practical applications.

\section{ACKNOWLEDGMENTS}

I am grateful to Guy Dimonte, Dan Klem, Chuck Leith, Karnig Mikaelian, Oleg Schilling, and Pete Stry for helpful discussions, comments, and suggestions, to Chuck Leith and Pete Stry for sharing their unpublished results, to Guy Dimonte for supplying me with his experimental data in digital form, and to Guy Dimonte, Karnig Mikaelian, and Oleg Schilling for directing my attention to some of the relevant literature. This work was performed under the auspices of the U.S. Department of Energy by Lawrence Livermore National Laboratory under Contract No. W-7405-ENG-48.
[1] H. J. Kull, Phys. Rep. 206, 197 (1991).

[2] R. D. Richtmyer, Commun. Pure Appl. Math. 13, 297 (1960).

[3] E. E. Meshkov, Izv. Akad. Nauk SSSR Mekh. Zhidk. Gaza 4, 151 (1969) [Izv. Acad. Sci. USSR Fluid Dynamics 4, 101 (1969)].

[4] D. L. Youngs, Physica D 12, 32 (1984).

[5] K. I. Read, Physica D 12, 45 (1984).

[6] G. Dimonte and M. Schneider, Phys. Rev. E 54, 3740 (1996).

[7] P. A. Thompson, Compressible-Fluid Dynamics (McGrawHill, New York, 1972).

[8] C. E. Leith, Lawrence Livermore National Laboratory report, 1985 (unpublished).

[9] G. I. Barenblatt, in Nonlinear Dynamics and Turbulence, edited by G. I. Barenblatt, G. Iooss, and D. D. Joseph (Pitman, Boston, 1983), p. 48.

[10] D. L. Youngs, Laser Part. Beams 12, 725 (1994).

[11] G. Dimonte and M. Schneider, Phys. Plasmas 4, 4347 (1997).

[12] P. G. Saffman and D. I. Meiron, Phys. Fluids A 1, 1767 (1989).

[13] K. O. Mikaelian, Phys. Fluids A 3, 2625 (1991).

[14] U. Alon, D. Ofer, and D. Shvarts, in Proceedings of the Fifth International Workshop on Compressible Turbulent Mixing, Stony Brook, NY, 18-21 July 1995, edited by R. Young, J. Glimm, and B. Boston (World Scientific, Singapore, 1996), p. 1.

[15] D. L. Youngs, in Proceedings of the Sixth International Work- shop on the Physics of Compressible Turbulent Mixing, Marseille, France, 18-21 June 1997, edited by G. Jourdan and L. Houas (Institut Universitaire des Systèmes Thermiques Industriels, Marseille, 1997), p. 534.

[16] H. Goldstein, Classical Mechanics, 2nd ed. (Addison-Wesley, Reading, MA, 1980).

[17] J. Glimm, Q. Zhang, and D. H. Sharp, Phys. Fluids A 3, 1333 (1991).

[18] K. O. Mikaelian, Physica D 36, 343 (1989).

[19] K. O. Mikaelian, Phys. Fluids A 2, 592 (1990).

[20] G. Dimonte, C. E. Frerking, and M. Schneider, Phys. Rev. Lett. 74, 4855 (1995).

[21] U. Alon, J. Hecht, D. Ofer, and D. Shvarts, Phys. Rev. Lett. 74, 534 (1995).

[22] H. Tennekes and J. L. Lumley, A First Course in Turbulence (MIT Press, Cambridge, MA, 1972).

[23] R. Clift, J. R. Grace, and M. E. Weber, Bubbles, Drops, and Particles (Academic, New York, 1978).

[24] J. C. V. Hansom et al., Laser Part. Beams 8, 51 (1990).

[25] K. O. Mikaelian, Phys. Rev. Lett. 80, 508 (1998).

[26] P. E. Stry (private communication).

[27] G. Dimonte (private communication).

[28] J. W. Jacobs and J. M. Sheeley, Phys. Fluids 8, 405 (1996).

[29] K. A. Meyer and P. J. Blewett, Phys. Fluids 15, 753 (1972).

[30] K. O. Mikaelian, Phys. Rev. Lett. 71, 2903 (1993).

[31] K. O. Mikaelian, Phys. Fluids 6, 356 (1994).

[32] K. O. Mikaelian, Phys. Rev. Lett. 73, 3177 (1994). 